\title{
Three-Dimensional Reconstruction of Extravascular Matrix Patterns and Blood Vessels in Human Uveal Melanoma Tissue: Techniques and Preliminary Findings
}

\author{
Xue Chen, ${ }^{1}$ Zhuming Ai, ${ }^{2}$ Mary Rasmussen, ${ }^{2}$ Peter Bajcsy, ${ }^{3}$ Loretta Auvil, ${ }^{3}$ Michael Welge, ${ }^{3}$ \\ Lu Leach, ${ }^{1}$ Sumalee Vangveeravong, ${ }^{1}$ Andrew J. Maniotis, ${ }^{1}$ and Robert Folberg ${ }^{1}$
}

\begin{abstract}
Purpose. Looping patterns rich in laminin are present in tissue samples of primary aggressive human uveal melanomas and their metastases. Because these extravascular patterns connect to blood vessels and transmit fluid in vitro and in vivo, the three-dimensional configuration of these patterns has been the subject of considerable speculation. In the current study, methods were devised to describe the three-dimensional configuration of looping extravascular matrix patterns in archival human uveal melanoma tissue.
\end{abstract}

Methods. Twenty-five serial $4-\mu \mathrm{m}$-thick sections from primary uveal melanoma tissue were labeled with fluorescence-tagged laminin and examined by confocal microscopy to generate a Z-series within each 4 - $\mu$ m-thick section. The $z$-series from each section was stacked using an immersive three-dimensional environment (ImmersaDesk; Fakespace, Kitchener, Ontario, Canada) to allow for precise alignment and compensation for distortion artifact.

RESUlts. Extravascular matrix patterns that appeared to form loops in two dimensions were shown to represent thin wrappings around branching and twisting cylindrical groupings of melanoma cells. Blood vessels joined with some of these laminin-positive cylindrical wrappings.

Conclusions. In this preliminary study, periodic acid-Schiff (PAS)-positive laminin-rich looping patterns in two-dimensional tissue sections appear to outline cylindrical branching packets of melanoma cells rather than spheroidal nests. The conduction of fluid through this extravascular system may provide a novel delivery system for contrast and diagnostic agents. (Invest Ophthalmol Vis Sci. 2003;44:2834-2840) DOI: 10.1167/iovs.02-1333

$\mathrm{T}$ here is a strong association between death in metastatic uveal melanoma and the presence of looping and crosslinked patterns positive for periodic-acid Schiff (PAS) in histo-

From the ${ }^{1}$ Department of Pathology and the ${ }^{2}$ Virtual Reality in Medicine Laboratory of the School of Biomedical and Health Information Sciences, University of Illinois at Chicago, Chicago, Illinois; and the ${ }^{3}$ Automated Learning Group, National Center for Supercomputing Applications, the University of Illinois at Urbana-Champaign, Champaign, Illinois.

Supported by Grants R01 EY10457 (RF) and Core Grant EY10792 (Department of Ophthalmology and Visual Sciences) from the National Eye Institute, Bethesda, Maryland.

Submitted for publication December 30, 2002; revised March 4, 2003; accepted March 11, 2003.

Disclosure: X. Chen, None; Z. Ai, None; M. Rasmussen, None; P. Bajcsy, None; L. Auvil, None; M. Welge, None; L. Leach, None; S. Vangveeravong, None; A.J. Maniotis, None; R. Folberg, None

The publication costs of this article were defrayed in part by page charge payment. This article must therefore be marked "advertise$m e n t "$ in accordance with 18 U.S.C. $\$ 1734$ solely to indicate this fact.

Corresponding author: Robert Folberg, Department of Pathology (MC 847), University of Illinois at Chicago, 1819 W. Polk Street 446 CMW, Chicago, IL 60612; rfolberg@uic.edu. logic sections of primary choroidal and ciliary body melanomas treated by enucleation. ${ }^{1}$ Looping patterns are detected in up to $88 \%$ of primary tumors of patients who died of metastatic uveal melanoma, and these patterns are present in sites of metastasis by uveal melanoma. ${ }^{2}$ Several laboratories ${ }^{3-6}$ have determined that looping patterns are independent prognostic indicators of death due to metastatic melanoma.

These patterns are generated in vitro by aggressive uveal melanoma cells without participation of endothelial cells and fibroblasts. ${ }^{7,8}$ In human primary uveal melanoma tissue, these patterns are composed ultrastructurally of a sandwich of electron-dense material on either side of tumor cells. ${ }^{8}$ Although these patterns have been shown to transport fluid in vitro, ${ }^{7,8}$ in animal models, ${ }^{9,10}$ and in human tissue samples, ${ }^{8}$ the patterns are clearly not vessels ultrastructurally. ${ }^{8}$ Furthermore, the patterns are not rich in type I collagen ultrastructurally, nor do they contain fibroblasts-additional evidence that is contrary to the notion that these patterns might represent fibrovascular septa. ${ }^{8}$ Several groups have now shown that these PAS-positive patterns are rich in laminin. $8,9,11-13$

The three-dimensional (3-D) configuration of extravascular matrix patterns that form back-to-back closed loops in twodimensional (2-D) tissue sections is unclear and highly controversial. ${ }^{14}$ Clay models suggested that looping extravascular matrix patterns form scaffolds around clusters of tumor cells. ${ }^{15}$ McDonald et al. ${ }^{14}$ used a computer simulation to suggest that these patterns envelope spheroids. However, the actual 3-D configuration of looping extravascular matrix patterns has never been demonstrated. Because these patterns conduct fluid in vivo, the distribution of diagnostic contrast agents and therapeutic agents through tumors that contain extravascular matrix patterns may be considerably different from that supplied by blood vessels alone. ${ }^{16}$

The distribution of blood in tumor tissue is often studied by using cast corrosions. ${ }^{17-19}$ After injecting latex or plastic into the circulation, tissue is digested leaving a cast of the blood flow to be studied by scanning electron microscopy. This technique cannot be used to discriminate between blood in vessels and extravascular matrix patterns, because every portion of the cast would be assumed to represent perfusion of a blood vessel.

Investigators have used serial tissue sections to develop 3-D reconstructions of blood vessels. ${ }^{20-27}$ Methods developed for 3-D tissue reconstruction must provide for precise section-tosection alignment and permit correction for heterogeneous tissue distortions from section to section when using paraffinembedded material, ${ }^{28,29}$ missing sections, or sections containing artifacts. Recently, Brey et al. ${ }^{16}$ reconstructed blood vessels from serial 2-D, $6-\mu \mathrm{m}$-thick frozen sections manually and by automated algorithms (thus circumventing artifacts introduced by paraffin embedding), and generated accurate renderings of the microcirculation, from which measurements of surface areas were calculated.

Several considerations preclude applying the methods described by Brey et al. ${ }^{16}$ in serial frozen sections to the 3-D 
reconstruction of laminin-rich extravascular matrix patterns in human primary and metastatic uveal melanomas. First, access to frozen primary human uveal melanoma tissue is limited. There are very few tissue banks containing archival frozen uveal melanoma samples, and most of these samples are quite small. Because laminin-rich extravascular matrix patterns are distributed heterogeneously throughout uveal melanoma tissue ${ }^{30}$ it is difficult to ensure a priori that stored small frozen tissue samples of human uveal melanoma in tissue banks contain extravascular matrix patterns of interest. Moreover, the realization that vision-sparing treatment such as radiation therapy is associated with no worse mortality than that achieved by enucleation $^{31}$ may limit the number of enucleations performed in the future and access to fresh tissue for morphologic studies. Second, conventional laser scanning confocal microscopy (LSCM) reconstructions permit investigators to visualize only the exterior of reconstructed tissue elements. To determine whether sheets of laminin encircle tumor cell spheroids as hypothesized, ${ }^{14,15}$ it becomes important to visualize the interior of the laminin compartments in addition to their surfaces.

In 1994, Rummelt et al. ${ }^{32}$ reconstructed blood vessels in histologic sections of uveal melanoma by LSCM and demonstrated the ability to apply multiple labels to identify both tumor cells and blood vessels in a 3 -D perspective. ${ }^{32}$ This reconstruction of tumor blood vessels was performed on a single, thick, paraffin-embedded tissue sample, thus eliminating concern about the alignment of serial sections. Although this method eliminated concern about heterogeneous distortions in paraffin-embedded tissue from section to section, resolution by this method was limited by computational power: as the tissue section thickness increases, the distance between confocal scanning planes in the $z$-series also increases, to avoid overwhelming the computational capacities of conventional confocal microscopy workstations. This results in a thick reconstruction with limited resolution. Moreover, the penetration of antibodies into very thick paraffin-embedded tumor sections may be uneven, making it difficult for investigators to discriminate between the absence of a signal and a lack of complete penetration of the antibody of interest.

We therefore designed a novel approach to tissue reconstruction on archival paraffin-embedded tissue by combining LSCM on serial tissue sections with reconstruction in an immersive stereo 3-D environment ${ }^{33}$ that has been used in biomedical visualization for drug design to visualize molecules and their receptors interactively ${ }^{34}$ and by radiologists to visualize complex interactions of various tissue surfaces. ${ }^{35}$ This technique permits correction for alignment and tissue distortions by applying warping algorithms on volumetric serial sections. In addition, the technology permits the researcher to dissect tissue digitally, to view the interior of objects of interest. After demonstrating that these novel techniques could be used to reconstruct blood vessels in paraffin-embedded tissue samples of retinoblastoma, a highly angiogenic tumor, we reconstructed extravascular matrix patterns from primary human uveal melanoma tissue.

\section{Materials ANd Methods}

\section{Immunohistochemistry}

Formalin-fixed, paraffin-embedded retinoblastoma and uveal melanoma tissue samples were sectioned at $4 \mu \mathrm{m}$ thickness. The use of archival human tissue in this study was approved by the Institutional Review Board of the University of Illinois at Chicago and was performed according to the tenets of the Declaration of Helsinki.

Slides were deparaffinized in xylene and rehydrated through a decreasing ethanol gradient. Slides were rinsed in distilled water followed by antigen unmasking using a $10 \times$ concentrated retrieval solu- tion (Target Retrieval Solution; Dako, Carpinteria, CA) according to the manufacturer's instructions and then rinsed in phosphate-buffered saline (PBS) for 5 minutes. For the demonstration of endothelium in fixed tissue of retinoblastoma, tissue sections were stained for CD31 (mouse monoclonal anti-human endothelial antibody to platelet- endothelial cell adhesion molecule [PECAM]-1; clone JC/70A; DAKO), and the reaction product was detected by diaminobenzidine (DAB). A preliminary study in our laboratory comparing CD34 with CD31 showed that $\mathrm{CD} 31$ consistently produces a stronger and more easily identifiable reaction product than $\mathrm{CD} 34$ in retinoblastoma tissue.

For the demonstration of laminin in human retinoblastoma and human uveal melanoma tissues by fluorescence and laser-scanning confocal microscopy, tissue sections were prepared as just described. Slides were then incubated with monoclonal mouse anti-laminin antibody (L8271, clone LAM 89; Sigma-Aldrich, St. Louis, MO) at a dilution titer of 1:200 for 30 minutes at room temperature. Slides were rinsed in protein-blocking solution (Dako) for 10 minutes, followed by detection with fluorescence-conjugated goat anti-mouse IgG (Alexa Fluor 488; Molecular Probes, Eugene, OR) for 30 minutes at a dilution of 1:400. Slides were rinsed in buffer then mounted in aqueous mounting medium (Faramount; Dako)

For the simultaneous detection of CD34 and laminin in human primary uveal melanoma tissue, slides were prepared as described earlier and then incubated with monoclonal mouse anti-human CD34 (QBend10; Dako) at a dilution of 1:40 for 30 minutes and placed in protein-blocking solution (Dako) for 10 minutes. Antibody binding was detected with the fluorescence-conjugated goat anti-mouse IgG for 30 minutes at a dilution titer of 1:400. After 10 minutes of protein blocking, slides were incubated with polyclonal rabbit anti-laminin antibody (Sigma-Aldrich) at a dilution titer of 1:200 for 30 minutes at room temperature. After protein blocking for 10 minutes, antibody binding was detected with fluorescence-conjugated goat anti-rabbit antibody (Alexa Fluor 594; Molecular Probes) for 30 minutes at a dilution titer of $1: 400$. Slides were rinsed in buffer and mounted in aqueous mounting medium (Faramount; Dako)

The simultaneous detection of laminin and $\mathrm{S} 100$ protein in primary human uveal melanoma tissue was conducted as described, with the following substitutions: a mouse monoclonal anti-laminin antibody (clone LAM-89; Sigma-Aldrich) at a dilution titer of 1:200 detected with fluorescence-conjugated goat anti-mouse IgG at a dilution of 1:400 and polyclonal rabbit antibody to $\$ 100$ protein (Dako) detected with fluorescence-conjugated goat anti-rabbit IgG at a dilution titer of 1:400. The simultaneous detection of laminin and fibrinogen in primary human uveal melanoma tissue was performed as described previously, ${ }^{8}$ using the following reagents: a mouse monoclonal anti-laminin antibody (clone LAM-89; Sigma) at a dilution titer of 1:200 detected with fluorescence-conjugated goat anti-mouse $\operatorname{IgG}$ at a dilution of 1:400 and polyclonal rabbit antibody to human fibrinogen (Dako) at a dilution titer of 1:1000 detected with fluorescence-conjugated goat anti-rabbit IgG at a dilution titer of 1:400. For all staining procedures, secondary antibody was omitted in negative control experiments.

\section{Fluorescence Microscopy}

Immunohistochemically double-labeled preparations (CD34-laminin and $\$ 100$ protein-laminin) were examined using a microscope equipped for fluorescence (BX40; Olympus America, Melville, NY). Digital images were captured (MagnaFire camera; Optronics, Goleta, CA) separately for each chromogen (in the case of multiple labels), and the images were merged with the accompanying software (MagnaFire software, ver. 2; Optronics).

\section{Laser Scanning Confocal Microscopy}

All histologic serial sections were examined with a laser scanning confocal microscope (LSM 510; Carl Zeiss Meditech, Thornwood, NY) using the $20 \times$ objective. Images were stored in laser scanning microscope format and converted on computer (LSM Image Browser software, ver. 2.50.0929; Carl Zeiss Meditech, Jena, Germany) into tagged 


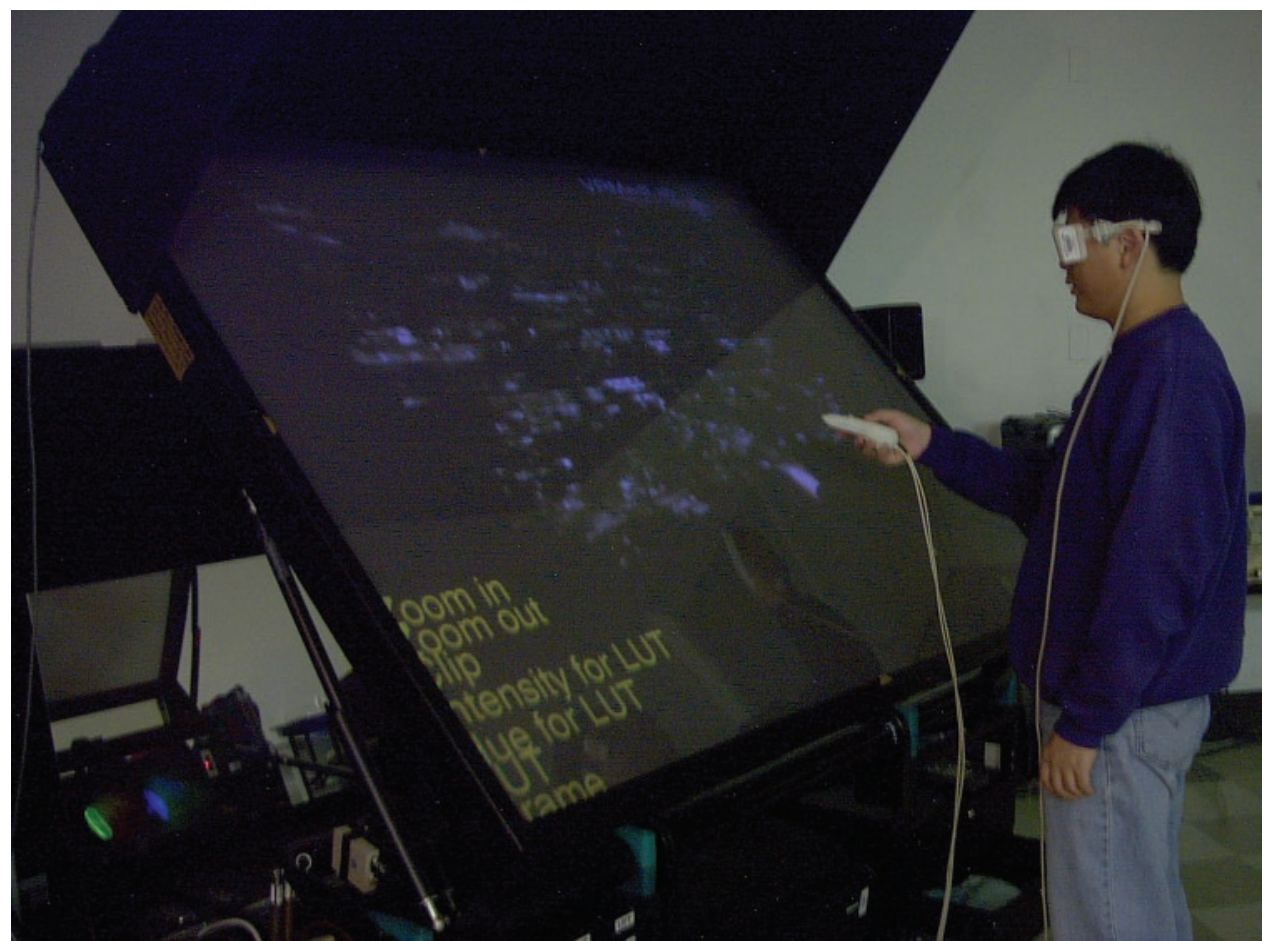

Figure 1. Using the ImmersaDesk (Fakespace, Kitchener, Ontario, Canada). The investigator is holding the "wand" by which he can manipulate the image on the screen, which is the reconstructed data set used to create Figure 3G. The positionally sensitive goggles worn by the investigator not only facilitate 3-D visualization but also permit the investigator to appear to move through the tissue by moving from side-to-side or by tilting the body in different planes. Additional goggles may be used by ancillary investigators to experience the same 3-D data set visualized by the investigator controlling motion with the positionally sensitive goggles. information file format (TIFF). For the 3-D reconstruction of blood vessels in retinoblastoma from $4-\mu \mathrm{m}$ sections stained with laminin with signal detection by immunofluorescence as described earlier, a $z$-stack of images of 20 components with $0.2-\mu \mathrm{m}$ intervals were obtained from five serial sections. Images for the 3-D reconstruction of two vessels in retinoblastoma tissue were obtained by this method. To reconstruct extravascular matrix patterns in primary human uveal melanoma tissue from $4-\mu \mathrm{m}$ sections stained with laminin with signal detection by immunofluorescence, as described earlier, a $z$-stack of eight components with $0.5-\mu \mathrm{m}$ intervals were obtained from each of 25 serial sections. Nine areas from two different primary uveal melanomas with extravascular matrix patterns were examined in this fashion.

\section{Three-Dimensional Immersive Visualization}

To perform, view, and manipulate the 3-D reconstructions, we also used ImmersaDesk ${ }^{33}$ (Fakespace, Kitchener, Ontario, Canada) which is a single-screen, portable appliance based on the $\mathrm{CAVE}^{36}$ virtual reality system developed by the Electronic Visualization laboratory (EVL) at the University of Illinois at Chicago. ImmersaDesk ${ }^{33}$ available in imageanalysis facilities at many research universities, offers the following advantages to investigators: the ability of multiple researchers to view data simultaneously, stereo vision, a wide viewing angle, and the ability to present different 3-D aspects of the image depending on the perspective of a designated investigator. Figure 1 illustrates the use of the ImmersaDesk $^{33}$ for viewing and manipulating the reconstructions developed from serial LSCM stacks. Investigators are equipped with lightweight goggles that contain shutter glasses made of transparent LCD panes that are blackened separately for each eye by applying a voltage that switches in rapid sequence between two views of the image, one for each eye. One goggle set also contains a device to track head position, which allows the computer to present data relevant to the investigator's position (e.g., as the investigator leans forward or to the side, he or she appears to travel through the reconstructed tissue). A wireless mouse-like device, a wand, permits the investigator to manipulate the reconstruction by turning it or slicing layers from the image to view internal structure.

\section{Three-Dimensional Reconstructions}

We designed a novel reconstruction strategy. After performing LSCM on each serial section to generate serial volumetric data, we then stacked the 3-D volumes in an immersive stereo environment. In Figure 2 , the subject of interest is a tilted cylinder. In aligning 2-D sections, one might arrive at a reconstruction that is a straight cylinder, rather than one that is tilted. However, in aligning images that have volumetric depth (bottom row), one matches alignment of the bottom image of the $z$-series from layer 1 with the top image from the Z-series of the next layer, layer 2, repeating this step until all images are aligned. Alignment is performed in an immersive 3-D environment (ImmersaDesk $^{37}$; Fakespace). In this example, manual alignment would not err in recreating a cylinder that is upright and would instead reconstruct the tilted cylinder correctly. If the object of interest in the bottom of the $z$-series of layer 1 does not match the top image in the next layer, the distortion, perhaps introduced by tissue processing, is corrected by applying a warping algorithm. A 3-D nonlinear warping algorithm is used to restore the distortion after the initial manual alignment. We use automated image reconstruction (AIR) software ${ }^{38}$ to apply a 2-D nonlinear warping algorithm to match the bottom image of the $z$-series from layer 1 with the top image from the $z$-series of layer 2, resulting in a 2-D $n$th order polynomial transformation (available at http://
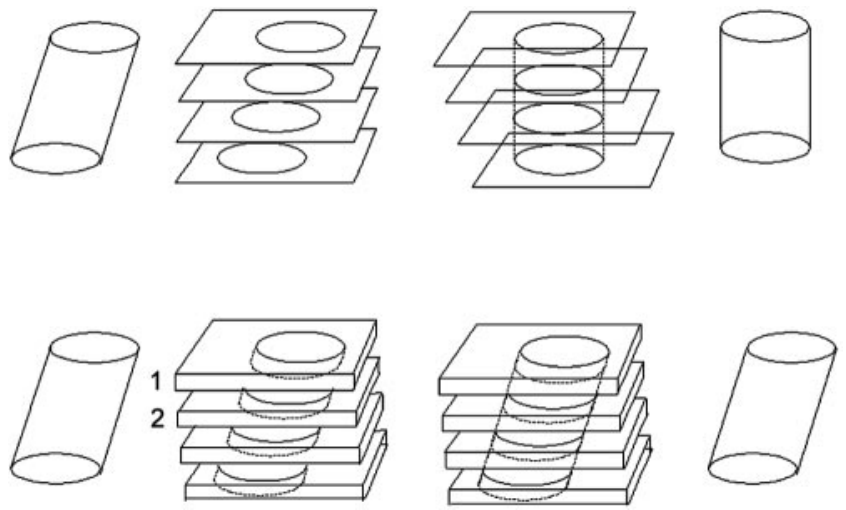

FIGURE 2. Comparison of a reconstruction performed by stacking 2-D images $(t o p)$ versus a reconstruction in an immersive stereo environment, performed by stacking 3-D volumes (bottom). The adjacent planes appear separated from one another in these sketches for illustration purposes only. 
128.97.134.164/AIR5/2Dnonlinear.html) which is expanded to 3-D ( $n$ +1 )th order (available at http://128.97.134.164/AIR5/3-Dnonlinear. html). With the known boundary conditions and some reasonable assumptions, all the coefficients in the $(n+1)$ th-order polynomial can be determined. With this nonlinear transformation, layer 1 can be warped to connect to layer 2 smoothly.

Video clips of the investigator interaction with the reconstructed dataset (see Appendix) were prepared by recording a session with the ImmersaDesk on a computer (model O2 with MediaRecorder software; SGI, Mountain View, CA). A window of $640 \times 480$ pixels was outlined on the ImmersaDesk display and was captured at 10 frames/sec (QuickTime format, Apple Computer, Cupertino, CA). Video-clips were then compressed and converted to avi format for replay on computer (Windows MediaPlayer; Microsoft, Bellevue, WA).

\section{Results}

Retinoblastoma is known to be a highly angiogenic tumor. ${ }^{39}$ To compare a baseline 3-D reconstruction of tumor vessels against which extravascular matrix patterns might be compared, tissue sections of retinoblastoma were first stained with CD31 to demonstrate blood vessels (Fig. 3A; blood vessels in retinoblastoma stained consistently weaker with CD34 than CD31 in trial studies; data not shown). Stains for laminin also labeled these vessels as expected (Fig. 3B). Three-dimensional reconstructions of two blood vessels in retinoblastoma were generated by the methods described earlier (Fig. 3C). Conventional 3-D reconstructions of LSCM stacks generated images only of the exterior of the object of interest. To demonstrate the use of the immersive stereo to digitally dissect tissue planes, we removed the upper wall of the blood vessel in Fig. $3 \mathrm{C}$ and rotated the image to reveal the entire length of the lining of the inferior wall of the vessel (Fig. 3D). Thus, the immersive stereo environment permits investigators to visualize the interiors of objects of interest.

Figures $3 \mathrm{E}-\mathrm{H}$ are representative of the nine reconstructions of extravascular matrix patterns performed on primary human uveal melanoma tissue. Figure $3 \mathrm{E}$ shows a 4 - $\mu$ m-thick tissue section of primary human uveal melanoma double labeled for laminin (red) and CD34 (green), with a paucity of CD34positive blood vessels. As shown previously, ${ }^{8}$ the looping laminin-positive extravascular matrix patterns are not stained with $\mathrm{CD} 34$. Figure $3 \mathrm{~F}$ is a section adjacent to $3 \mathrm{E}$ and is double labeled with laminin (green) and S100 protein (red) to illustrate the fact that melanoma cells ( $\mathrm{S} 100$ positive) occupy the tissue spaces within the looping laminin-positive patterns.

Figure $3 \mathrm{G}$ is a $3-\mathrm{D}$ reconstruction of the primary uveal melanoma tissue presented in $3 \mathrm{E}$ and $3 \mathrm{~F}$. The tissue sample illustrated in $3 G$ was labeled with laminin only (which identifies both blood vessels and the extravascular patterned matrix). In Figure 3G, branching blood vessels are easily identified (arrows), corresponding to the CD34-positive vessels in the adjacent double-labeled section (Fig. 3E). What appear to be back-to-back loops of laminin in the 2-D plane (Fig. 3E, red; Fig. $3 \mathrm{~F}$, green) are actually the outer linings of cylinders that wrap around what appear to be empty spaces, because the tissue section is labeled only for laminin (these "spaces" are actually filled with S100-positive melanoma cells that do not label with laminin; see Fig. 3F). Although the tissue reconstructed in Figure 3G was stained only with laminin (which detects both blood vessels and the extravascular patterned matrix), it is clear that the cylinders are not themselves blood vessels, because they do not stain with the endothelial cell marker CD34 (3E). The laminin-positive cylinders are not regularly oriented topically. For example, the space in Figure $3 G$ that is marked with the single asterisk would appear in a 2-D section to be a loop, but in the 3-D environment, it is a cross-section of a cylinder that extends in Figure $3 G$ from right to left and posteriorly into the section.

In video clip 1 (see Appendix), a recording of the investigator interaction with the 3-D reconstruction presented in Fig. $3 G$, the reconstructed tissue is rotated from side to side, clearly illustrating the cylindrical laminin-positive outline surrounding the tumor marked with the single asterisk. However, in the last frame of video clip 1, note that there was an oval "defect" in the otherwise confluent laminin lining. This defect, like all "space" in this reconstruction, is populated by S100-positive tumor cells. Parenthetically, a cross-section of tissue through a defect in the wall of a cylinder would generate an arc instead of a loop, an observation consistent with the hierarchical relationships of patterns in histologic 2-D section planes. ${ }^{40}$

In video clip 2 (see Appendix), the investigator not only rotates the reconstruction shown in static representation from right to left (Fig. 3G), but also tilts the reconstruction backward. With this maneuver, the space (actually occupied by S100 protein-positive melanoma cells), marked with the plus sign $(+)$ in Fig. 3G, is shown to be subdivided by branching laminin-positive septa (note the last frame of this video clip), thus showing that this cylinder of tumor cells divides or branches.

Figure $3 \mathrm{H}$ is a 3-D reconstruction from another area of primary uveal melanoma performed under immersive visualization. The tissue in Figure $3 \mathrm{H}$, double labeled with laminin (green) and fibrinogen (red), shows colocalization of fibrinogen (yellow) to the laminin-rich extravascular matrix patterns, consistent with our previously observation based on 2-D tissue sections. ${ }^{8}$ The colocalization of fibrinogen, a surrogate marker for plasma, to these laminin-rich patterns is consistent with the flow of fluid through these patterns in animal models after intravenous injection ${ }^{9,10,41}$ and the observation of red blood cells within the patterns in human tissue samples.

\section{Discussion}

These preliminary reconstructions of extravascular matrix patterns in primary uveal melanoma tissue suggest that the backto-back loops and networks that are prognostically significant in this tumor may be composed in part of laminin-positive wrappings around cylinders of uveal melanoma cells that may branch and divide. These observations are entirely consistent with recently published in vitro observations ${ }^{8}$ indicating that looping patterns in human uveal melanoma tissues are unlikely to represent spheroidal aggregates of aggressive tumor cells.

If the cylindrical orientation of laminin is discovered to be a consistent feature of these patterns in repeated studies of multiple tumors, the irregular orientation of the extravascular matrix patterns would have several important clinical implications. First, there is evidence from in vitro studies, ${ }^{7,8}$ animal models, ${ }^{10}$ and human tissue samples ${ }^{8}$ that these extravascular matrix patterns are perfusable. Future refinements of the techniques illustrated in this article will allow for tissue reconstructions on multiple fluorescence wavelengths to detect simultaneously the laminin in cylindrical extravascular matrix patterns, the $\mathrm{S} 100$ positivity in melanoma cells filling the laminin-positive cylinders, and the presence of plasma by intravenous fluorescent tracer in an animal model. ${ }^{42}$ If the cylindrical laminin-positive extravascular matrix is richly perfused, as suggested by studies of 2-D tissue sections, then extravascular matrix patterns may provide a novel distribution pathway for diagnostic contrast media ${ }^{43-45}$ and novel therapeutic agents.

Second, there is preliminary evidence that it is possible to detect the presence of prognostically significant extravascular matrix patterns in the eyes of patients by power-spectrum analysis of raw radiofrequency data obtained from noninvasive 
FiguRE 3. (A) Retinoblastoma tissue stained for CD31 to demonstrate blood vessels. Note red blood cells inside the vessels. (B) Retinoblastoma tissue: two blood vessels are labeled with laminin. (C, D) Threedimensional reconstruction of blood vessels in (B) from a $z$-series generated by LSCM and prepared using immersive stereo visualization techniques. (C) With conventional LSCM reconstruction techniques, we cannot visualize the interior lining of the lower portion of the vessel (*). However, in the 3-D immersive environment, we digitally "removed" the upper vessel wall above the plane of the arrow in (C); and, after rotating the image downward (D), we could visualize the entire length of the lining of the lower portion of the vessel $(*$ the same reference point as in the micrograph in C). (E) Primary human uveal tissue melanoma is labeled with CD34 for endothelium (green) and laminin (red). There are very few endothelial lined blood vessels ( $a r$ rows) within the laminin-positive extravascular matrix. Note also that the patterns themselves are not lined by endothelium (i.e., they are CD34 negative). (F) Primary human uveal melanoma in which the melanoma cells are stained for $\$ 100$ protein (red) and the extravascular patterned matrix is demonstrated by staining with laminin (green). (G) This is a 3 -D reconstruction generated in an immersive environment of a primary human uveal melanoma stained for laminin from sections adjacent to that illustrated in $(\mathbf{F})$. Blood vessels (arrows) are identified. Laminin-positive cylinders appear to wrap around empty spaces, but in reality, these empty spaces are packed with S100-positive tumor cells (F). In the 3-D environment, it becomes apparent that laminin is outlining cylindrical structures, not spheroidal structures. Note that the cylinder marked with the single asterisk (*) appears to be oriented obliquely, whereas the space marked with the plus $(+)$ appears oriented perpendicular to the plane of view. (H) This 3-D reconstruction is taken from an area of a primary uveal melanoma different from the one illustrated in (G) and is double labeled for laminin (green) and fibrinogen $(r e d)$. Note the colocalization of fibrinogen to the laminin, consistent with previous observations $^{8}$ made from the labeling of 2-D sections. Because a narrower interval was used with the retinoblastoma tissue, the resolution of the blood vessels in the retinoblastoma tissue is 2.5 times greater in the $z$-axis than the resolution in the $z$-axis for the uveal melanoma reconstructions. Resolution of the images in $(\mathbf{C})$ and $(\mathbf{D})=1.75 \times 1.75 \times 5$ pixels $/ \mu \mathrm{m}$; resolution of the image in $(\mathbf{G})$ is $1.4 \times 1.4 \times 2$ pixels $/ \mu \mathrm{m}$ with the frame depicted in $(\mathbf{G})$ measuring $365.7 \times 365.7 \times 100 \mu \mathrm{m}$. Resolution of the image in $(\mathbf{H})$ is $1.38 \times 1.38 \times 2$ pixels/ $\mu \mathrm{m}$, equivalent to $512 \times 512 \times$ $192 \mu \mathrm{m}$.

ultrasonography of the tumor, ${ }^{46}$ independent of any possible perfusion characteristics of these patterns. These encouraging data, however, were based on the assumption that the looping patterns represent spheroidal nests of tumor cells. If additional reconstructions confirm the results that looping patterns are instead anisotropic arrangements of cylinders of tumor cells wrapped by laminin-rich matrix, as this preliminary study suggests, then different analytical algorithms can be applied to ultrasonic raw radiofrequency data to obtain even more accurate correlations between noninvasive ultrasonographic imag- 
ing and histologic properties of the tumor. With more patients electing vision-sparing treatment rather than enucleation, the ability to obtain prognostic information by noninvasive substitutes for biopsy- either through angiographic detection of perfused extravascular matrix patterns or by the ultrasonographic organization of melanoma tissue into bending cylinders wrapped in laminin-rich matrix-may be useful in allowing ophthalmologists and oncologists to stratify patients into low- and high-risk groups.

Although the present study illustrates the feasibility of developing 3-D reconstructions of extravascular matrix patterns from archival tissue samples, there is considerable room for further methodological refinements. The strategy applied in this preliminary study included the manual alignment of serial tissue volumes rather than serial sections and the manual correction for inhomogeneous tissue distortions. It is also possible to automate these steps and to correct for translational, rotational, and shear misalignments to eliminate any subjective interpretation of data. Moreover, in future data sets, missing sections can be handled with a method similar to 3-D warping by constructing a section with the bottom image of the $z$-series from layer 1. Using the 3-D warping algorithm describe earlier, this section is warped and inserted into the gap. Finally, given the fact that there are only a few markers that discriminate between blood vessels and extravascular matrix patterns, it may be helpful to develop quantifiable numeric characteristics that topologically distinguish between tubular, branching blood vessels, and the larger cylinder-wrappings of lamininpositive, fluid-conducting matrix.

With these more advanced analytical tools, it will be possible to study a large library of archival tissue samples of primary and metastatic human uveal melanoma, to study the variability of extravascular matrix pattern topology, and to compare perfusion of fluid in three dimensions through extravascular matrix patterns with perfusion through tumor blood vessels in an established animal model. ${ }^{42}$

\section{References}

1. Rummelt V, Folberg R, Rummelt C, et al. Microcirculation architecture of melanocytic nevi and malignant melanomas of the ciliary body and choroid. a comparative histopathologic and ultrastructural study. Opbthalmology. 1994;101:718-727.

2. Rummelt V, Mehaffey MG, Campbell RJ, et al. Microcirculation architecture of metastases from primary ciliary body and choroidal melanomas. Am J Opbthalmol. 1998;126:303-305.

3. Sakamoto T, Sakamoto M, Yoshikawa $H$, et al. Histologic findings and prognosis of uveal malignant melanoma in Japanese patients. Am J Opbthalmol. 1996;121:276-283.

4. McLean IW, Keefe KS, Burnier MN. Uveal melanoma: comparison of the prognostic value of fibrovascular loops, mean of the ten largest nucleoli, cell type and tumor size. Opbthalmology. 1997; 104:777-780.

5. Seregard S, Spangberg B, Juul C, Oskarsson M. Prognostic accuracy of the mean of the largest nucleoli, vascular patterns, and PC-10 in posterior uveal melanoma. Ophthalmology. 1998;105:485-491.

6. Makitie T, Summanen P, Tarkannen A, Kivela T. Microvascular loops and networks as prognostic indicators in choroidal and ciliary body melanomas. J Nat Cancer Inst. 1999;91:359-367.

7. Maniotis AJ, Folberg R, Hess A, et al. Vascular channel formation by human melanoma cells in vivo and in vitro: vasculogenic mimicry. Am J Patbol. 1999;155:739-752.

8. Maniotis AJ, Chen X, Garcia C, et al. Control of melanoma morphogenesis, endothelial survival, and perfusion by extracellular matrix. Lab Invest. 2002;82:1031-1043.

9. Potgens AJG, van Altena MC, Lubsen NH, Ruiter DJ, de Waal RMW. Analysis of the tumor vasculature and metastatic behavior of xenografts of human melanoma cell lines transfected with vascular permeability factor. Am J Patbol. 1996;148:1203-1217.
10. Clarijs R, Otte-Holler I, Ruiter DJ, de Waal RM. Presence of a fluid-conducting meshwork in xenografted cutaneous and primary human uveal melanoma. Invest Ophthalmol Vis Sci. 2002;43:912918.

11. Seftor REB, Seftor EA, Koshikawa N, et al. Cooperative interactions of laminin $5 \gamma 2$ chain, matrix metalloproteinase-2, and membrane type-1-matrix/metalloproteinase are required for mimicry of embryonic vasculogenesis by aggressive melanoma. Cancer Res. 2001;61:6322-6327

12. Sood AK, Seftor EA, Fletcher MS, et al. Molecular determinants of ovarian cancer plasticity. Am J Patbol. 2001;158:1279-1288.

13. Thies A, Mangold U, Moll I, Schumacher U. PAS-positive loops and networks as a prognostic indicator in cutaneous malignant melanoma. J Patbol. 2001;195:537-542.

14. McDonald DM, Munn L, Jain RK. Vasculogenic mimicry: how convincing, how novel, and how significant? Am J Patbol. 2000; 156:383-388.

15. Barinaga M. A face-off over tumor blood supply. Science. 2000; 287:783-785.

16. Brey EM, King TW, Johnston C, McIntire LV, Reece GP, Patrick CW. A technique for quantitative three-dimensional analysis of microvascular structure. Microvasc Res. 2002;63:279-294.

17. Dvorak HF, Nagy JA, Dvorak JT, Dvorak AM. Identification and characterization of the blood vessels of solid tumors that are leaky to circulating macromolecules. Am J Patbol. 1988;133:95-109.

18. Konerding MA, Miodonski AJ, Lametschwandtner A. Microvascular corrosion casting in the study of tumor vascularity: a review. Scanning Microsc. 1995;9:1233-1243.

19. Konerding MA, Malkusch W, Klapthor B, et al. Evidence for characteristic vascular patterns in solid tumours: quantitative studies using corrosion casts. Br J Cancer. 1999;80:724-732.

20. Furusato M, Wakui S, Suzuki M, et al. Three-dimensional ultrastructural distribution of cytoplasmic interdigitation between endothelium and pericyte of capillary in human granulation tissue by serial section reconstruction method. J Electron Microsc. 1990;39:8691.

21. Wakui S, Furusato M, Itoh T, et al. Tumour angiogenesis in prostatic carcinoma with and without bone marrow metastasis: a morphometric study. J Patbol. 1992;168:257-262.

22. Furusato M, Wakui S, Sasaki H, Ito K, Ushigome S. Tumour angiogenesis in latent prostatic carcinoma. BrJ Cancer. 1994;70:12441246.

23. Perez-Atayde AR, Sallan SE, Tedrow U, Connors S, Allred E, Folkman J. Spectrum of tumor angiogenesis in the bone marrow of children with acute lymphoblastic leukemia. Am J Patbol. 1997; 150:815-821.

24. Kay PA, Robb RA, Bostwick DG. Prostate cancer microvessels: a novel method for three-dimensional reconstruction and analysis. Prostate. 1998;37:270-277.

25. Antiga L, Ene-Iordache B, Remuzzi G, Remuzzi A. Automatic generation of glomerular capillary topological organization. Microvasc Res. 2001;62:346-354.

26. Patan S, Munn LL, Tanda S, Roberge S, Jain RK, Jones RC. Vascular morphogenesis and remodeling in a model of tissue repair: blood vessel formation and growth in the ovarian pedicle after ovariectomy. Circ Res. 2001;89:723-731.

27. Patan S, Tanda S, Roberge S, Jones RC, Jain RK, Munn LL. Vascular morphogenesis and remodeling in a human tumor xenograft: blood vessel formation and growth after ovariectomy and tumor implantation. Circ Res. 2001;89:732-739.

28. Jones AS, Milthorpe BK, Howlett CR. Measurement of microtomyinduced section distortion and its correction for 3-dimensional histological reconstructions. Cytometry. 1994;15:95-105.

29. Mega MS, Chen SS, Thompson PM, et al. Mapping histology to metabolism: coregistration of stained whole-brain sections to premortem PET in Alzheimer's disease. Neuroimage. 1997;5:147153.

30. Folberg R, Fleck M, Mehaffey MG, et al. Mapping prognostically significant vascular patterns in ciliary body and choroidal melanomas. Patbol Oncol Res. 1996;2:229-236.

31. Diener-West M, Earle JD, Fine SL, et al. The COMS randomized trial of iodine 125 brachytherapy for choroidal melanoma, III: Initial mortality findings. Arch Ophthalmol. 2001;119:969-982. 
32. Rummelt V, Gardner LM, Folberg R, et al. Three-dimensional relationships between tumor cells and microcirculation using double cyanine-immunolabeling, laser scanning confocal microscopy and computer-assisted reconstruction: an alternative to cast corrosion preparations. J Histochem Cytochem. 1994;42:681-686.

33. Czernuszenko M, Pape D, Sandin D, DeFanti T, Dawe G, Brown M. The ImmersaDesk and infinity wall projection-based virtual reality displays. Comp Grapbics. 1997;31:46-49.

34. Nagata $H$, Mizushima $H$, Tanaka $H$. Concept and prototype of protein-ligand docking simulator with force feedback technology. Bioinformatics. 2002;18:140-146.

35. Hopper KD, Iyriboz AT, Wise SW, Neuman JD, Mauger DT, Kasales CJ. Mucosal detail at CT virtual reality: surface versus volume rendering. Radiology. 2000;214:517-522.

36. Cruz-Niera C, Sandin DJ, DeFanti T. Surround-screen projectionbased virtual reality: the design and implementation of the CAVE. Proc Siggraph. 1993;93:135-142.

37. Johnson CR. Computational bioimaging for medical diagnosis and treatment. Comm Assoc Comput Machinery. 2001;43:74-76.

38. Woods RP, Cherry SR, Mazziotta JC. Rapid automated algorithm for aligning and reslicing PET images. J Comput Assist Tomogr. 1992; 16:620-633.

39. Pe'er J, Neufeld M, Baras M, Gnessin H, Itin A, Keshet E. Rubeosis iridis in retinoblastoma: histologic findings and the possible role of vascular endothelial growth factor in its induction. Opbthalmology. 1997; 104:1251-1258.

40. Folberg R, Chen X, Boldt HC, et al. Microcirculation patterns other than loops and networks in choroidal and ciliary body melanomas. Ophthalmology. 2000;108:996-1001.

41. Shirakawa K, Kobayashi H, Heike Y, et al. Hemodynamics in Vasculogenic mimicry and angiogenesis of inflammatory breast cancer xenograft. Cancer Res. 2002;62:560-566.

42. Mueller AJ, Maniotis AJ, Freeman WR, et al. An orthotopic model for human uveal melanoma in SCID mice. Microvasc Res. 2002; 64:207-213.

43. Mueller AJ, Bartsch DU, Folberg R, et al. Imaging the microvasculature of choroidal melanomas with confocal indocyanine green scanning laser ophthalmoscopy. Arch Opbthalmol. 1998;116:3139.
44. Mueller AJ, Freeman WR, Folberg R, et al. Evaluation of microvascularization pattern visibility in human choroidal melanomas: comparison of confocal fluorescein with indocyanine green angiography. Graefes Arch Clin Exp Opbthalmol. 1999;237:448-456.

45. Mueller AJ, Schaller U, Freeman W, Folberg R, Kampik A. Complex microcirculation patterns detected by confocal Indocyanine green angiography predict time to growth of small choroidal melanocytic tumors: MuSIC-Report II. Ophthalmology. 2002;109:22072214.

46. Silverman RH, Folberg R, Boldt HC, et al. Correlation of ultrasound parameter imaging with microcirculatory patterns in uveal melanomas. Ultrasound Med Biol. 1997;23:573-581.

\section{APPENDIX}

View the video clips described below at http://www.iovs.org/ cgi/content/full/44/7/2834/DC1.

\section{Video Clip 1}

The 3-D reconstruction from $3 \mathrm{G}$ is rotated in real time by the investigator. The image of the hand is the icon of the wand used to manipulate the image. As the entire reconstruction is rotated from side-to-side, the "space" encircled by laminin and marked with an asterisk in Figure 3G assumes a cylindrical shape. In reality, there is no space because S100-positive tumor cells occupy this compartment. Not staining for the tumor cells makes the 3-D distribution of laminin visible.

\section{Video Clip 2}

The 3-D reconstruction from Figure $3 \mathrm{G}$ is rotated in real time by the investigator. In this reconstruction, the investigator, wearing position-sensitive goggles (Fig. 1), has leaned forward toward the ImmersaDesk, rotating the reconstruction back. The image is then rotated from side-to-side with the wand as in video clip 1 . The "space" marked with the plus sign $(+)$ in Figure $3 G$ is now the center of attention. Note that what appears to be a clear space from front to back in Figure $3 G$ is actually subdivided by branching laminin-positive septa in this video clip. 